\title{
Compact SRF Accelerator applications: USPAS Lecture
}

\author{
Jayakar "Charles" Thangaraj , Fermilab
}




\section{Accelerators for industry}

- Accelerators for industrial applications:

- Modest energy: few MeVs - tens of MeV

- Modest and high power: tens of kW - hundreds of kW.

- Specific requirements:

- Simplicity

- Low cost

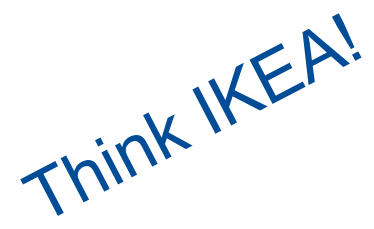

- Reliability

- Work in industrial environment (sometimes harsh)

- Easy to operate

- Small sizes

- High efficiency

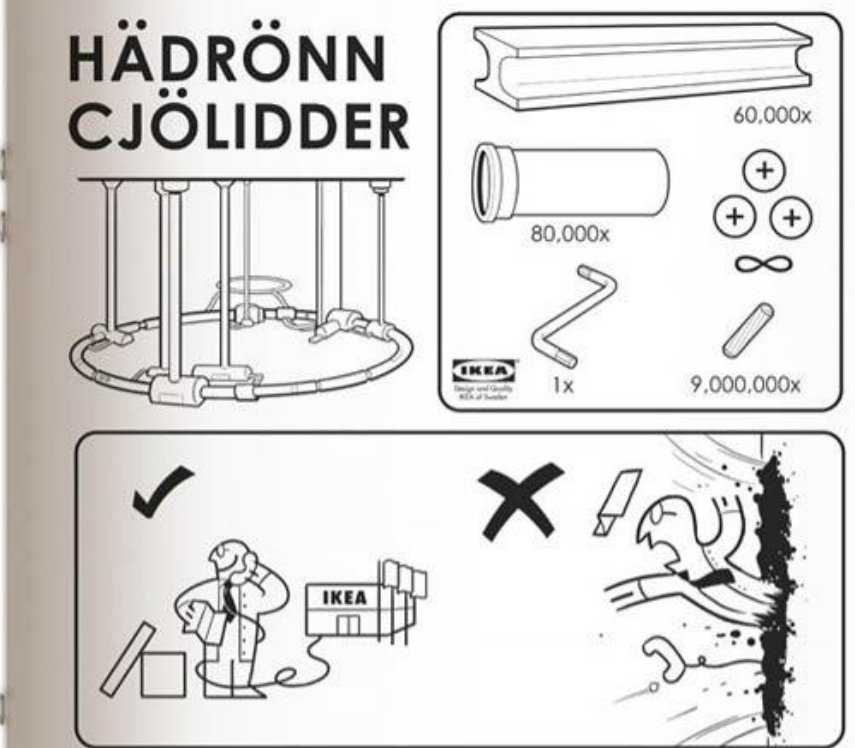

1.

\begin{tabular}{l|l}
2. \\
\hline
\end{tabular}
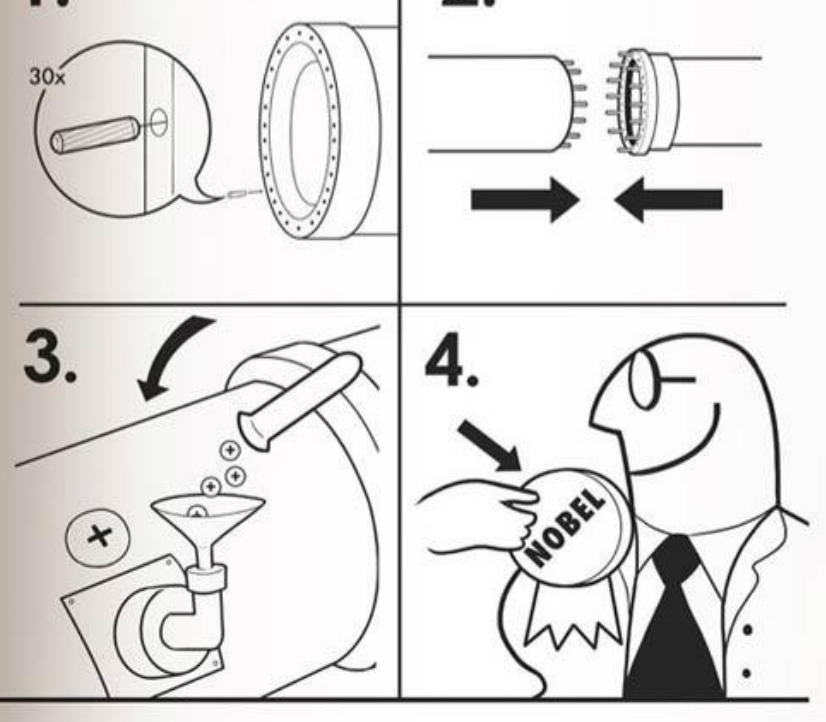


\section{Accelerators comes in several sizes and shapes.}

- Electrostatic (few keV - $10 \mathrm{MeV}$ ) - e.g. Dyanmitron, Cockroft-Walton, Pelletron

- Microtron - a cross of cyclotron but uses multi-pass

- Betatron - essentially a transformer but circular can reach several MeV's

- Rhodotron - recirculating through a coaxial cavity

- RF Linac (several MeV's) - normal conducting cavities

- Synchrotron

- Ion accelerators (different species)

A steady market 


\section{Commercial EB accelerator applications are vast}

- EB welding

- EB melting

- EB sterilization

- EB curing

- Non-destructive testing

- Medical imaging

- Cargo inspection 


\section{Current vs New Accelerator Technology}

- Bulk materials processing applications require multi-Mev energy for penetration or to generate x-rays and 100's of kW (or even MW) of beam power

- > few MeV accelerators are typically copper and RF driven

- Inherent losses limit efficiency (heat vs beam power) = ops cost

- Heat removal limits duty factor, gradient and average power $\rightarrow$ physically large "fixed" installations = CAPEX

\section{New Technology: Superconducting Radio Frequency (SRF)}

- High wall plug power efficiency (e.g. 75\%)

- Large fraction of the input power goes into beam

- High power \& efficiency enables new \$ 1 Billion class SRF-based science machines $\rightarrow$ driving large R\&D efforts at labs

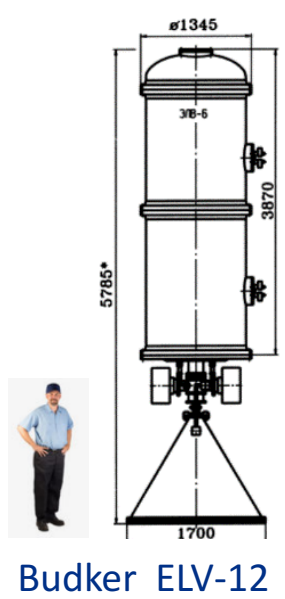

- Currently SRF-based science accelerators are huge with complex cryogenic refrigerators, cryomodules, etc. But this is changing!

- Recent SRF breakthroughs now enable a new class of compact, SRF-based industrial accelerators (lower CAPEX and OPS cost) 


\section{Superconducting Radio Frequency (SRF)}

$\sim$ All new high beam power accelerators for discovery science employ SRF

- Why?

- Because $\sim$ all RF power $\rightarrow$ beam power vs heating RF resonators

- SRF $\rightarrow$ Higher gradient, more energy per unit length

- But current SRF "science" accelerators are large and complex

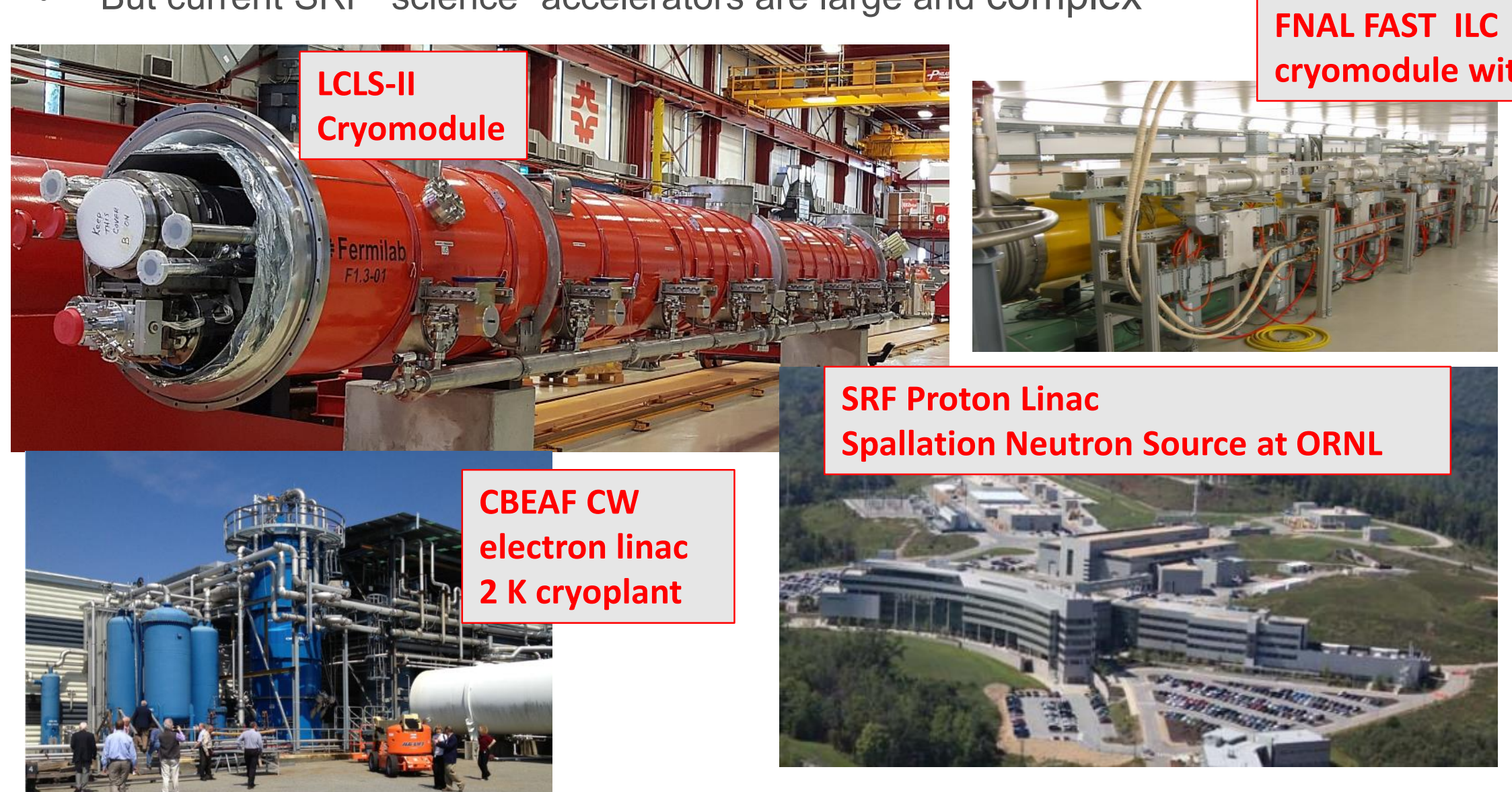




\section{Why superconducting}
Technology
Energy
Power
Issues/Potential
Room temperature
Few $\mathrm{MeV}$
Up to few hundred kW's
- Energy efficiency
- Heat loss
- Old(er) technology
Superconducting
$10 \mathrm{MeV}$
$100 \mathrm{~kW}-1+\mathrm{MW}$
- $\mathrm{CW}$
- Excellent energy efficiency
- Reliable, cutting-edge technology based on science machines (>1 \$B)
- Compact cryogenics 


\section{Ideas integrated into a simple SRF accelerator}

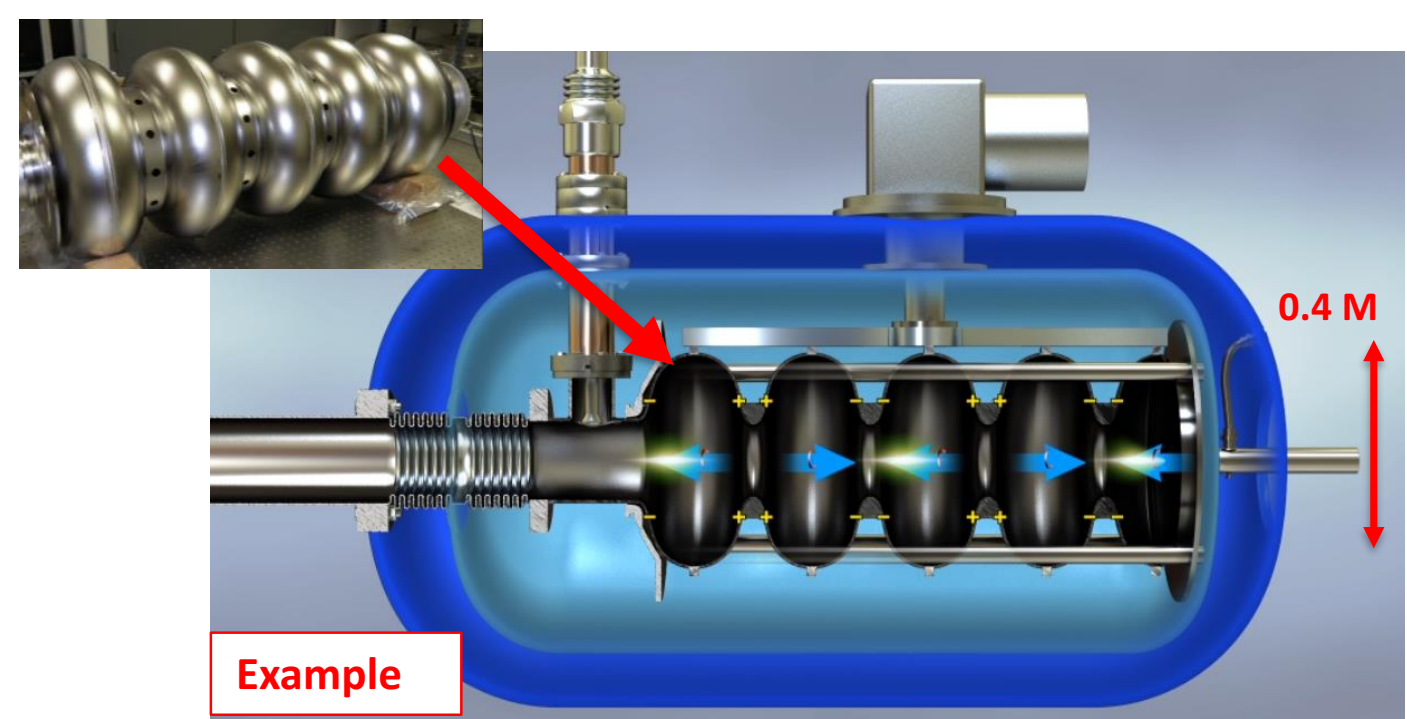

Final machine parameters

- Energy: $10 \mathrm{MeV}$

- Power: 250 kW - $1 \mathrm{MW}$

- Compact

- Simple, reliable

- Affordable

- $650 \mathrm{MHz}$ elliptical cavity (well understood from PIP-II)

- Modular design scales to MW class industrial applications 


\section{Recent SRF Technology Breakthroughs:}

- Higher temperature superconductors: $\mathrm{Nb}_{3} \mathrm{Sn}$ coated cavities dramatically lower cryogenic losses and allow higher operating temperatures (e.g. $4 \mathrm{~K}$ vs $1.8 \mathrm{~K}$ )

- Commercial Cryocoolers: new devices with higher capacity at $4 \mathrm{~K}$ enables turn-key cryogenic systems

- Conduction Cooling: possible with low cavity losses $\rightarrow$ dramatically simplifies cryostats (no Liquid Helium !)

- New RF Power technology: injection locked magnetrons allow phase/amplitude control at high efficiency and much lower cost per watt

- Integrated electron guns: reduce accelerator complexity

- Enable compact industrial SRF accelerators at low cost 


\section{Solicitation for advancing industrial accelerators}

- Dept. of Energy provided funding to develop novel accelerator designs to address need for industrial application in the energy and environment sectors

\begin{tabular}{|c|c|c|c|c|}
\hline & $\begin{array}{l}\text { Type } 1 \\
\text { Demo/Small } \\
\text { Scale }\end{array}$ & $\begin{array}{l}\text { Type } 2 \\
\text { Medium } \\
\text { Scale Low } \\
\text { Energy }\end{array}$ & $\begin{array}{l}\text { Type } 3 \\
\text { Medium Scale } \\
\text { High Energy }\end{array}$ & $\begin{array}{l}\text { Type } 4 \\
\text { Large Scale } \\
\text { High Energy }\end{array}$ \\
\hline $\begin{array}{l}\text { Example } \\
\text { Applications }\end{array}$ & $\begin{array}{l}\text { R\&D, } \\
\text { Sterilization, } \\
\text { industrial } \\
\text { effluent streams }\end{array}$ & $\begin{array}{l}\text { Flue Gas, } \\
\text { Waste water }\end{array}$ & $\begin{array}{l}\text { Wastewater, } \\
\text { sludge, medical } \\
\text { waste }\end{array}$ & $\begin{array}{l}\text { Sludge, Medical } \\
\text { waste, Env. } \\
\text { remediation }\end{array}$ \\
\hline $\begin{array}{l}\text { Electron Beam } \\
\text { Energy }\end{array}$ & $0.5-1.5 \mathrm{MeV}$ & $1-2 \mathrm{MeV}$ & $10 \mathrm{MeV}$ & $10 \mathrm{MeV}$ \\
\hline $\begin{array}{l}\text { Electron Beam } \\
\text { Power }(\mathrm{CW})\end{array}$ & $>0.5 \mathrm{MW}$ & $>1 \mathrm{MW}$ & $>1 \mathrm{MW}$ & $>10 \mathrm{MW}$ \\
\hline $\begin{array}{l}\text { Wallplug } \\
\text { Efficiency }\end{array}$ & $>50 \%$ & $>50 \%$ & $>50 \%$ & $>75 \%$ \\
\hline $\begin{array}{l}\text { Target Capital } \\
\text { Cost* }\end{array}$ & $<\$ 10 / W$ & $<\$ 10 / W$ & $<\$ 10 / W$ & $<\$ 5 / \mathrm{W}$ \\
\hline $\begin{array}{l}\text { Target Operating } \\
\text { Cost }\end{array}$ & $<1.0 \mathrm{M} \$ / \mathrm{yr}$ & $<1.5 \mathrm{M} \$ / \mathrm{yr}$ & $<1.5 \mathrm{M} \$ / \mathrm{yr}$ & $<12 \mathrm{M} \$ / \mathrm{yr}$ \\
\hline
\end{tabular}


$1 \mathrm{MeV}, 1 \mathrm{MW}$ SRF accelerator

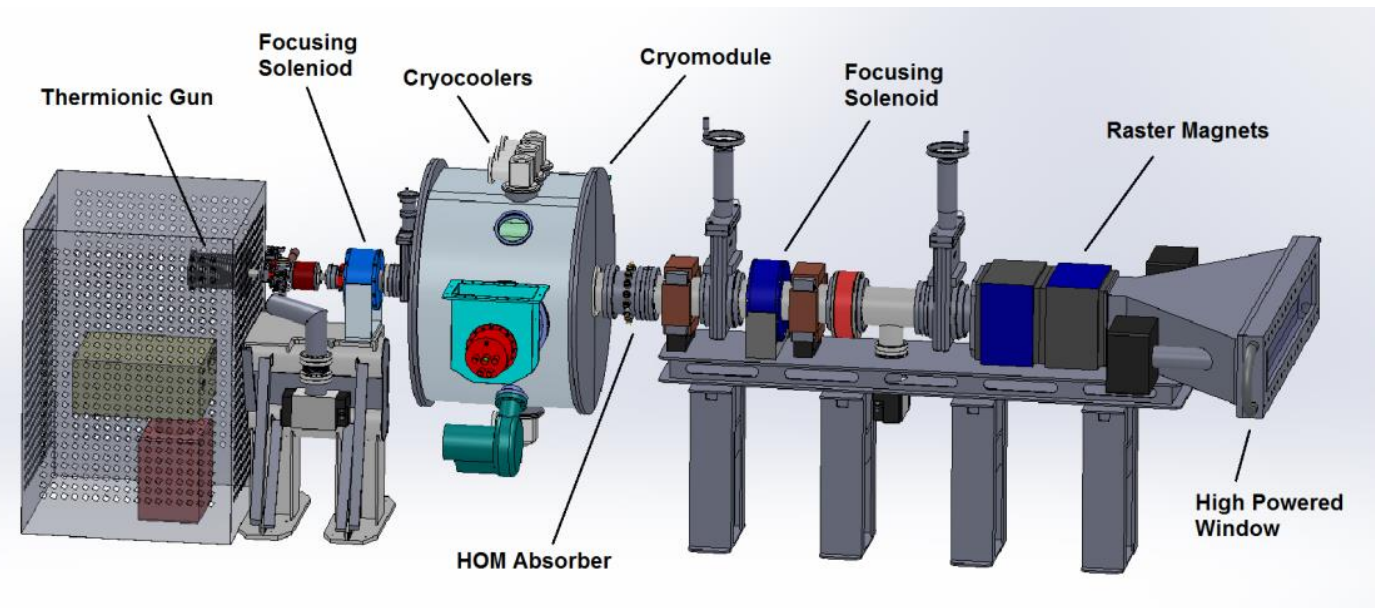

Jefferson Lab

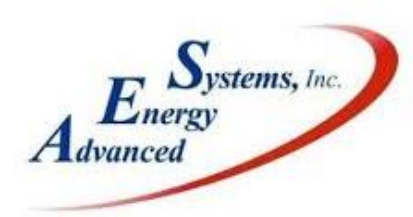

G. Ciovati, R. Rimmer, F. Hannon, J. Guo, F. Marhauser, V. Vylet

J. Rathke, T. Schultheiss

J. Anderson, B. Coriton, L. Holland, M. LeSher

[2] G. Ciovati et al., https://arxiv.org/abs/1802.08289

\section{$10 \mathrm{MeV}, 1 \mathrm{MW}$ SRF accelerator}

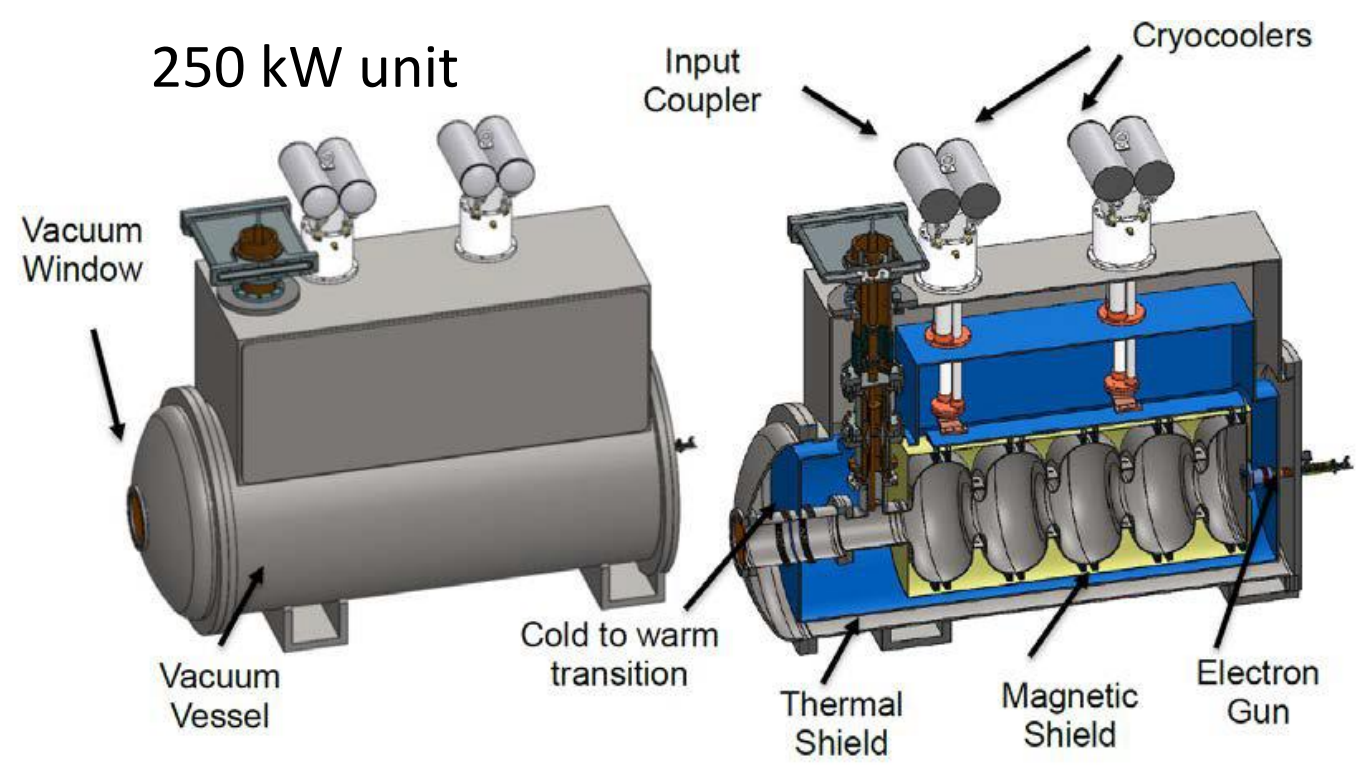

R. Kephart, V. Yakovlev, N. Solyak , I. Gonin , S. Kazakov , Fermilab T. Khabiboulline, O. Prokofiev, S. Posen

T. Kroc, C. Cooper, J. Thangaraj, R. Dhuley, M. Geelhoed

Northern Illinois

University

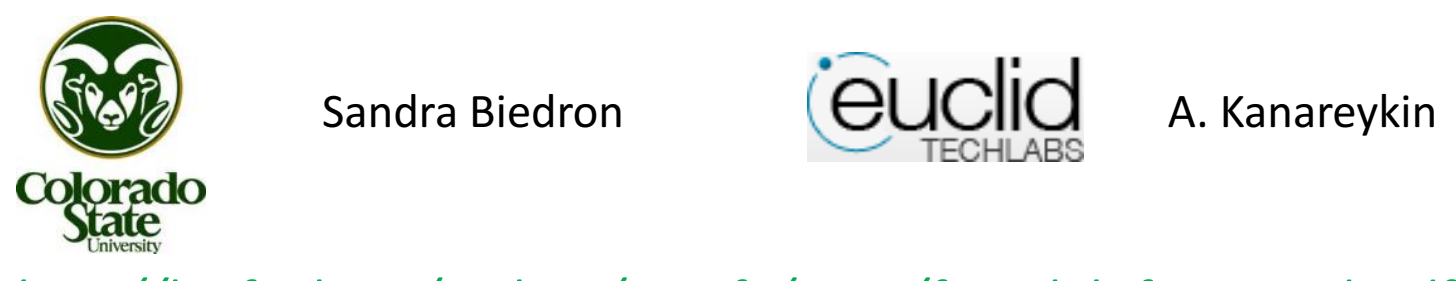

[3] http://Iss.fnal.gov/archive/test-fn/1000/fermilab-fn-1055-di.pdf 


\section{Facilities Layout}

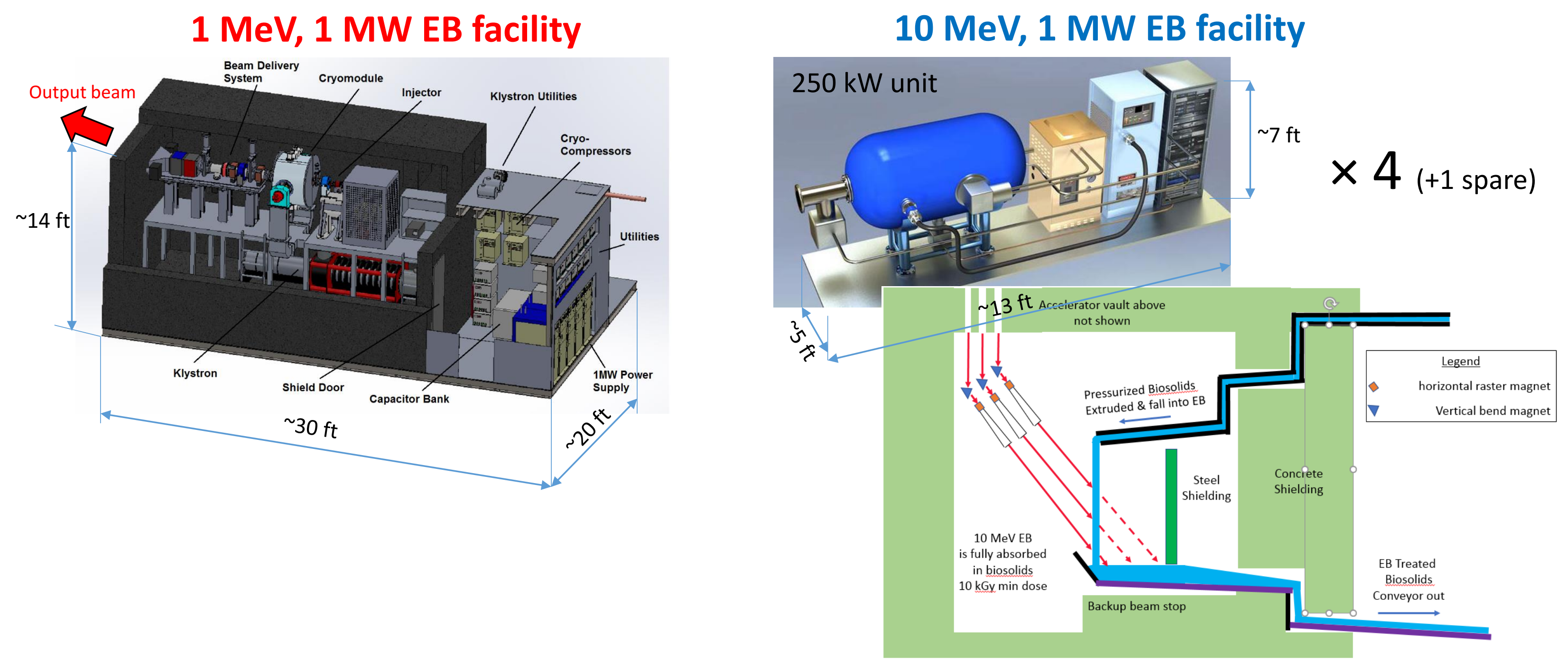




\section{New opportunities with compact industrial SRF-based accelerators}




\section{Future Accelerator Applications}

\section{Energy and Environment}

- Treat Municipal Waste \& Sludge

- Eliminate pathogens in sludge

- Destroy organics, pharmaceuticals in waste water

- In-situ environmental remediation

- Contaminated soils

- Spoils from dredging, etc

\section{Industrial and Security}

- Catalyze Chemical reactions to save time and energy

- In-situ cross-link of materials

- Improve pavement lifetime

- Instant cure coatings

- Medical sterilization without Co60

- Improved non-invasive inspection of cargo containers

These new applications need cost effective, energy efficient, high average power electron beams.

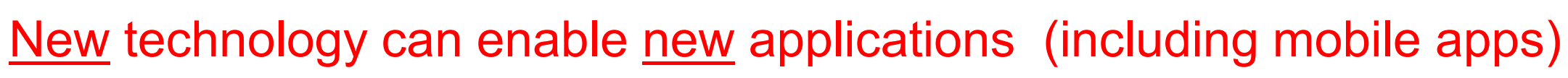




\section{Economics of SRF E-beam treatment}

\section{(acknowledgment to: Gianluigi Ciovati, JLab)}

Physics > Accelerator Physics

\section{Design of a cw, low energy, high power superconducting linac for environmental applications}

G. Ciovati, J. Anderson, B. Coriton, J. Guo, F. Hannon, L. Holland, M. LeSher, F. Marhauser, J. Rathke, R. Rimmer, T. Schultheiss, V. Vylet

(Submitted on 22 Feb 2018)

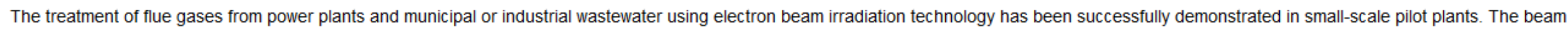

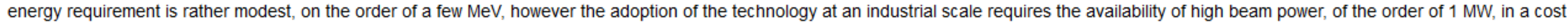

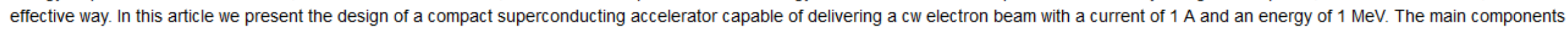

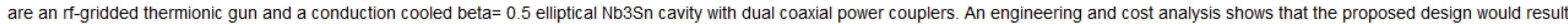
in a processing cost competitive with alternative treatment methods.

Subjects: Accelerator Physics (physics.acc-ph)

Cite as: arXiv:1802.08289 [physics.acc-ph]

(or arXiv:1802.08289v1 [physics.acc-ph] for this version) 


\section{Cost estimate for $1 \mathrm{MeV}, 1 \mathrm{MW}$ SRF EB facility}

\begin{tabular}{|l|l|}
\hline Capital Cost & \\
\hline SRF Accelerator & $\$ 4,500,000$ \\
\hline Infrastructure & $\$ 2,750,000$ \\
\hline Total & $\$ 7,250,000$ \\
\hline Investment (20\%) & $\$ 1,450,000$ \\
\hline Amortization(15yr @ 8\%) & $\$ 670 \mathrm{k} / \mathrm{yr}$ \\
\hline
\end{tabular}

\begin{tabular}{|l|l|}
\hline Operating Cost $(8,000$ hrs/yr) & \\
\hline Power & $\$ 159.2 / \mathrm{hr}$ \\
\hline Cooling water & None (air-cooled chillers) \\
\hline Maintenanceb) & $\$ 145 \mathrm{k} / \mathrm{yr}$ \\
\hline Total & $\$ 1,418,600 / \mathrm{yr}$ \\
\hline Total Cost (Capital + Op.) & $\mathbf{\$ 2 6 1 / \mathbf { h r }}$ \\
& $\mathbf{\$ 2 , 0 8 8 , 6 0 0 / y r}$
\end{tabular}

\section{Assumptions}

a) $2.274 \mathrm{MW}$ (Elec. Eff.: 42\%) @ \$0.07/kWh

b) $2 \%$ capital/year

c) No dedicated operator

Electric powe

Amortization

Maintenance

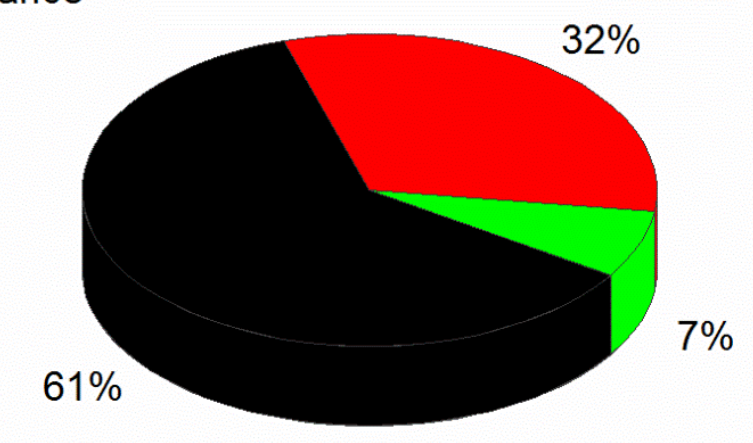




\section{Processing cost sensitivity to Design Parameters}
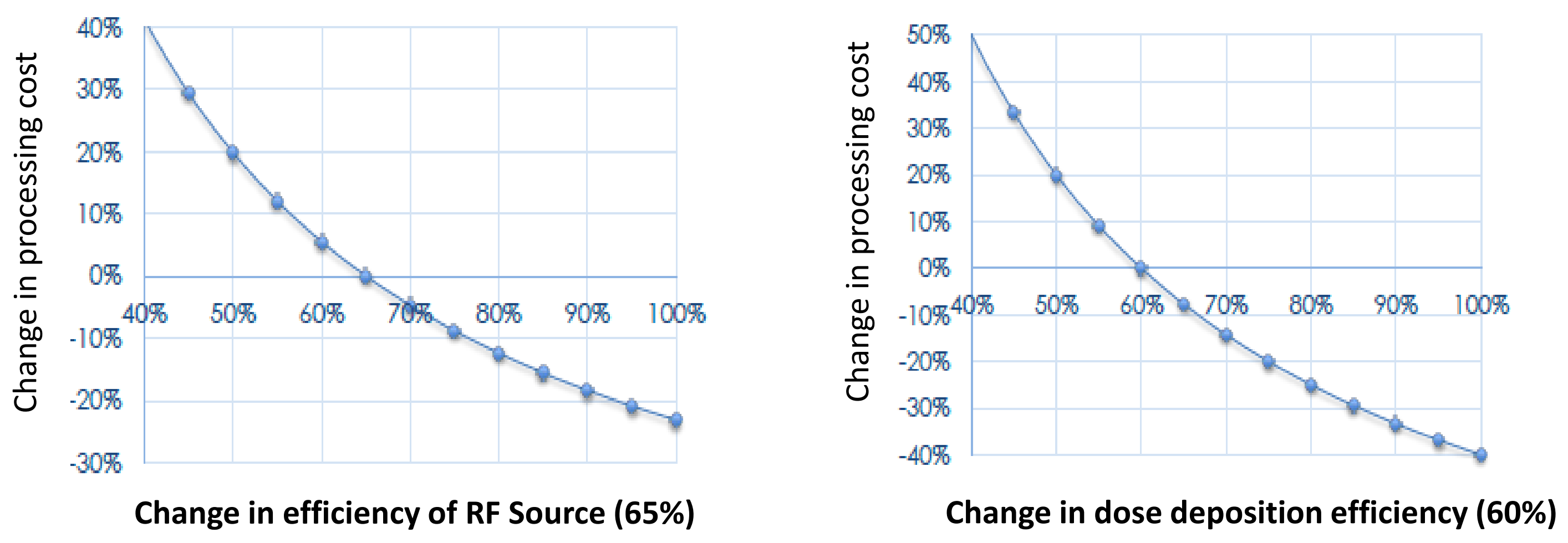

Current technology: klystron (65\%), IOT (70\%)

In development: magnetrons (90\%) 


\section{Processing cost sensitivity to Operation Parameters}
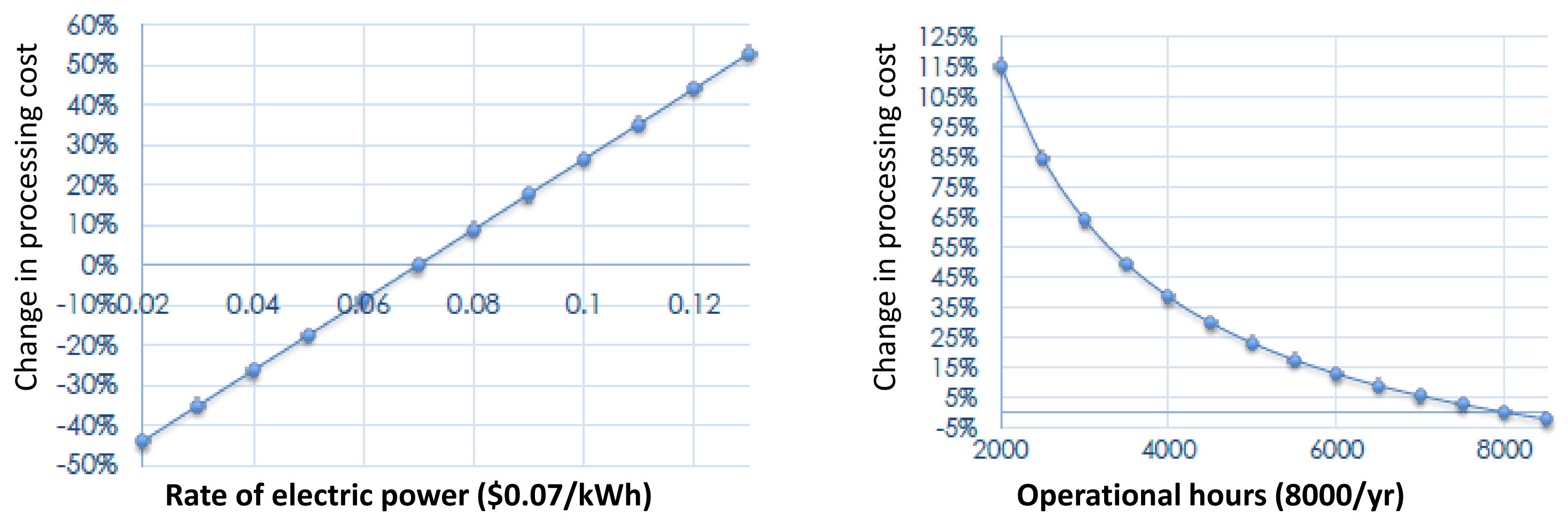


\section{Processing cost per Application}

\begin{tabular}{|l|c|c|c|} 
& \multicolumn{2}{|c|}{$1 \mathrm{MeV}, 1 \mathrm{MW}$} & $10 \mathrm{MeV}, 1 \mathrm{MW}$ \\
\hline & \multicolumn{2}{|c|}{ WASTEWATER } & SLUDGE \\
\hline Dose requirement & $1 \mathrm{kGy}$ & $4 \mathrm{kGy}$ & $10 \mathrm{kGy}$ \\
\hline \begin{tabular}{l|l} 
Processing cost \\
Cost of current technologies \\
(other than EB) $[4]$
\end{tabular} & $\$ 0.13 / \mathrm{m}^{3}(\$ 0.482 / \mathrm{kgal})$ & $\$ 0.51 / \mathrm{m}^{3}(\$ 1.93 / \mathrm{kgal})$ & $\$ 19.7 / \mathrm{dry}$ ton \\
\hline
\end{tabular}

\begin{tabular}{|l|c|c|c|}
\hline Daily Processed Volume & $\begin{array}{c}45,000 \mathrm{~m}^{3} \\
(11.9 \mathrm{Mgal})\end{array}$ & $\begin{array}{c}11,250 \mathrm{~m}^{3} \\
(3.0 \mathrm{Mgal})\end{array}$ & $\begin{array}{c}278 \text { dry ton (1.3 Mgal } \\
\text { with 25\% biosolid } \\
\text { waste) }\end{array}$ \\
\hline Required Flow Rate (gpm) & 9,050 & 2,260 & 984 \\
\hline Comments [4] & $\begin{array}{c}\text { Color, Odor, Coliform } \\
\text { bacteria removal }\end{array}$ & Kill $>99 \%$ of bacteria & $\begin{array}{c}\text { Inactivate some } \\
\text { radiation resistant } \\
\text { organisms }\end{array}$ \\
\hline
\end{tabular}




\section{Emerging application in pavement application}
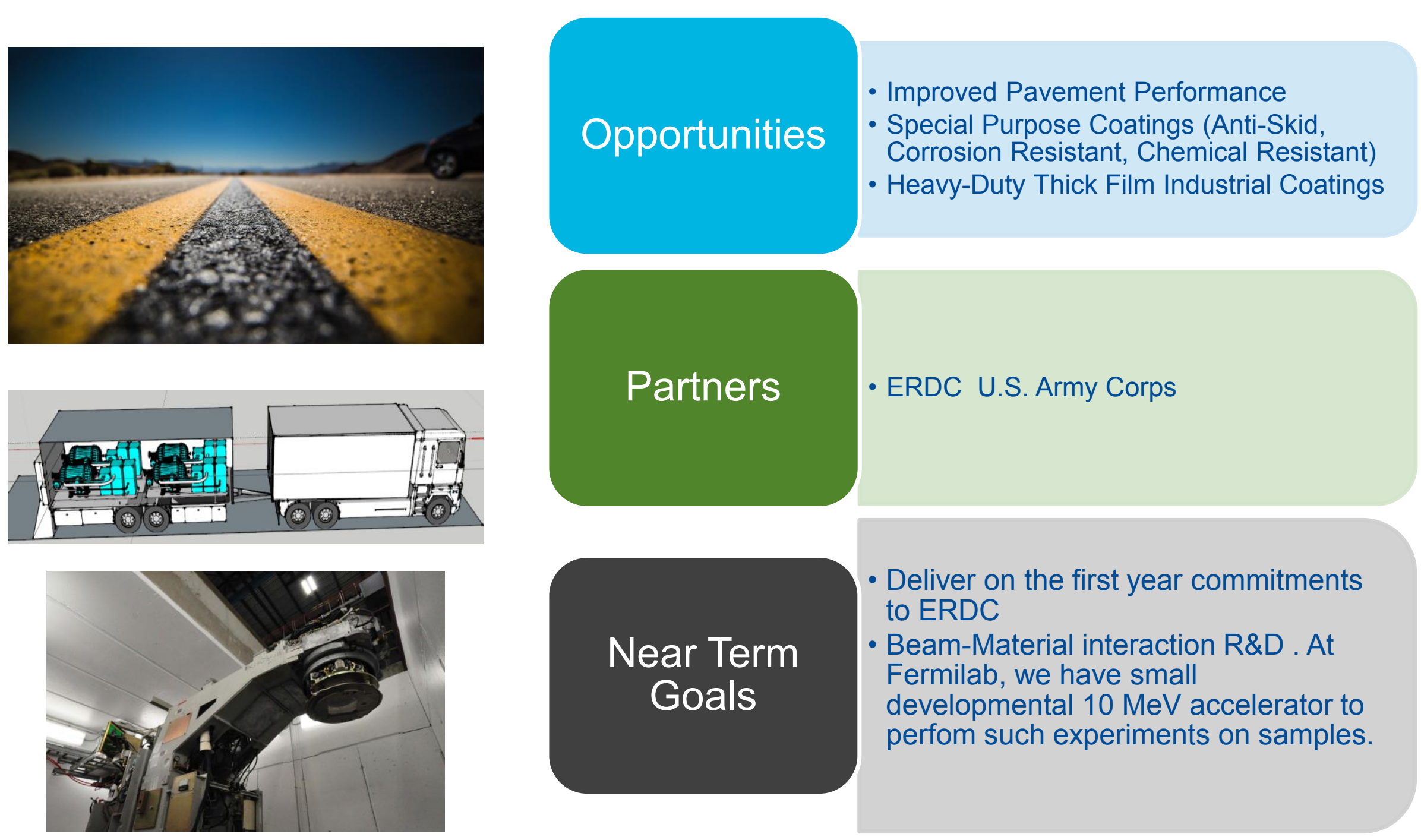

Deliver on the first year commitments to ERDC

Near Term

- Beam-Material interaction R\&D . At Fermilab, we have small

Goals

developmental $10 \mathrm{MeV}$ accelerator to perfom such experiments on samples. 


\section{Many emerging areas that SRF accelerators can add value}

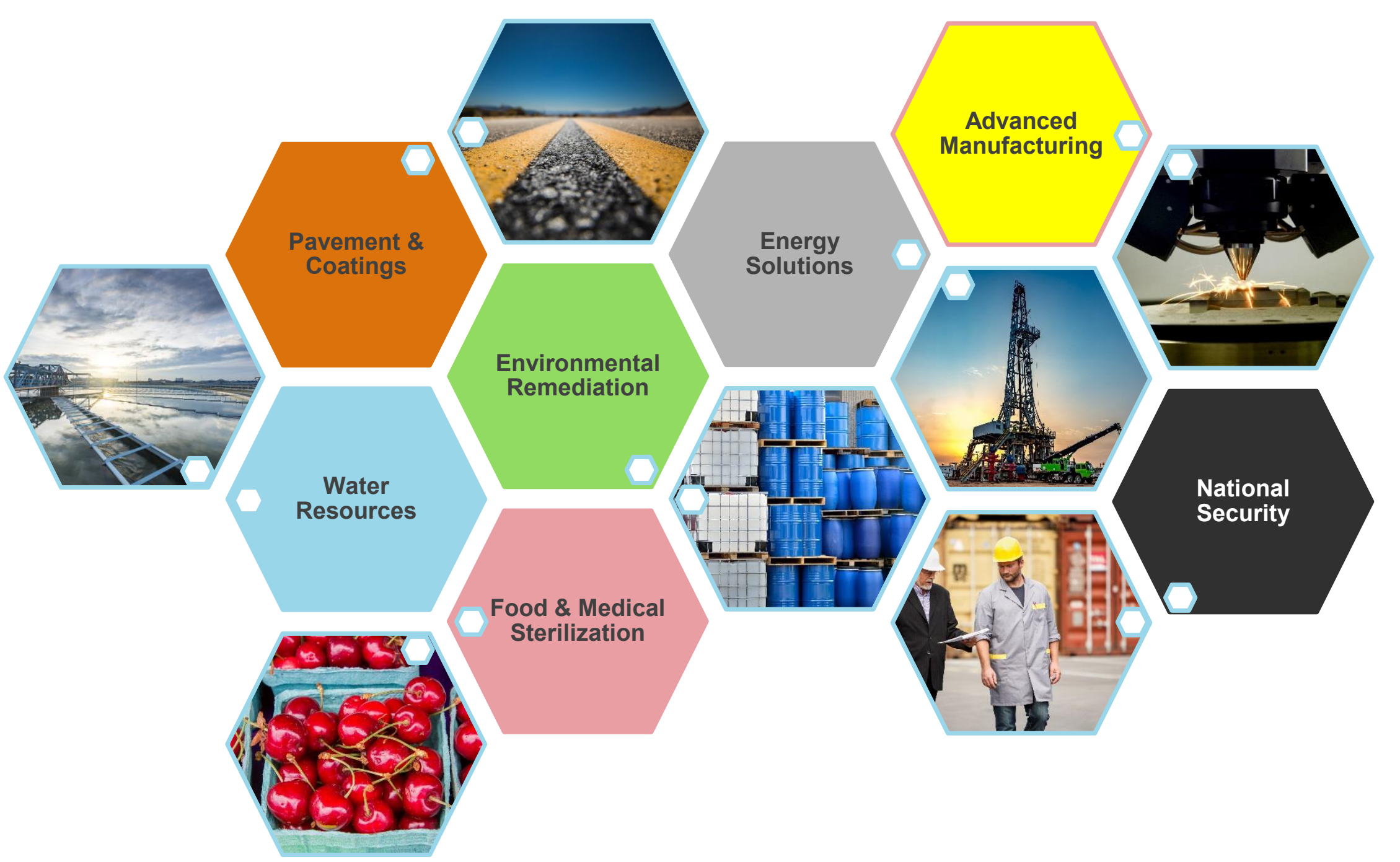




\section{Conclusions}

- The Illinois Accelerator Research Center at Fermilab is partnered with U.S. government agencies to create the first article of an entirely new class of industrial SRF-based electron accelerators that use no liquid cryogens

- Mobile, high energy, high power, high efficient electron accelerators can enable a variety of entirely new industrial applications

- Several applications may have enormous market potential

- If you are a student interested in working with Fermilab, talk to me for opportunities! I will be happy to help. 